\title{
Developing a communication strategy framework for the Southern Africa Regional Hunger \& Vulnerability Programme
}

Lydie Terblanche

\section{Abstract}

The Southern Africa Regional Hunger and Vulnerability Programme (RHVP) is a three-year programme of support for policy-makers concerned with food security, social protection, and vulnerability analysis in the SADC region. RHVP aims to improve food security at both national and regional levels in the SADC region by improving capacities of national governments to initiate or expand social protection programmes; strengthening and helping to institutionalise systems for vulnerability assessment and analysis in support of social protection policy; and supporting complementary investigative work to identify and improve understanding of new approaches to reducing hunger and vulnerability.

The components of the programme included: information and research, capacity building and training, commitment, influence, and policy change. The programme coordinators required assistance in order to formalise ideas and concepts into an actual working plan, in other words a communication strategy framework to determine communication needs, objectives, and goals.

*Lydie Terblanche lecturers part time in the Department of Communication and Information Studies, the Department of Agricultural Economics, the Centre for Sustainable Agriculture, and at DIMTEC (Disaster Management Training and Education Centre for Africa), at the University of the Free State.

\section{Background}

A strategy (plan of action) consists of techniques (methods) and tactics (rules) which are supportive of the given strategy. Moreover, any strategy should complement policy as well as the overall principle that underlies the whole programme.

Integral to the Regional Hunger and Vulnerability Programme (RHVP) is the dissemination of evidence and sharing of understanding emerging from ongoing research and action learning around alternatives to food aid. This communication strategy lays out a framework for dynamic communication activity involving a diverse range of stakeholders that will be driven by the RHVP Learning Network team and implemented and reviewed throughout the course of the programme.

The strategy outlines an approach for effective external communication arising out of the three core programme components - evidence building (ongoing research), capacity building, and influencing policy change. The communication strategy is located as part of the activities for the RHVP network, which communicate and advocate the outputs of the programme. The advocacy strategy is a more deliberate process of influencing those who make policy decisions, rather than general dissemination.

The strategy attempts to encourage an integrative approach i.e. - where communication vehicles are already established we contribute to them e.g.: Social Protection Newsletter or the Department of Agriculture's Food Security newsletter in Mozambique. The strategy also acknowledges that communication is not always one way and embraces the notion of 
dialogue and debate i.e. - covertly we will constantly push for recognition of alternatives to food aid but we will nevertheless be encouraging debate about emerging policy and practice across the board through, for example, radio discussions or learning events.

\section{The environment within which RHVP is operating}

With recurring food crises year on year, food security in the Southern Africa region is a very live issue. The programme needs to be in a position to communicate continually and effectively with a range of stakeholders in appropriate ways.

The external environment needs to be continually monitored and reviewed to ensure awareness:

of government policies and relevant food security activities in each of the six RHVP countries; of ongoing WFP policies and practices; of policy developments in international /regional organisations; of operational programme activities by international NGO's working in the 6 RHVP countries; and of international academic research addressing food security issues in the 6 RHVP countries.

At any time, RHVP needs to be positioned to respond to and engage in debate about food security within the region and to talk authoritatively about alternatives.

Purpose and objectives of the communication strategy

The purpose of the communication strategy is to inform stakeholders about RHVP and findings and lessons emerging from the programme's core themes:

achieving a better understanding of chronic vulnerability as a guide to policy priorities; vulnerability and assessment analysis (how to understand);

social protection (what does it mean, choices between alternative social protection approaches to reducing vulnerability to hunger and how they can be implemented in different contexts);

analysing food markets and regional trade; and

political and governance factors determining policy uptake.

The following steps are part of achieving the communication goals (Model adapted from Stone 1991:60 and Grunig \& Hunt 1984: 124-125):

Message

To create awareness: At this stage, the public may not be aware of RHVP.

Awareness

At this stage, the public becomes aware of the RHVP and its objectives. Normally awareness means a slight or definite uneasiness, but not a concrete definition or knowledge of the objective.

Knowledge 
Questions are asked, and discussions take place and RHVP and its goals become recognisable - given a "name," so to speak.

Understanding

During this stage, the first signs of a need for wanting change are observed.

Perception

The will to change can only be mobilised once a perception of the need for change is experienced.

Belief

Belief refers to the public's belief in a positive outcome. But this can only happen once

Attitude

changes. In this phase, thus, the will to change becomes mobilised.

Behaviour

The process is completed only once behaviour is changed.

Effective implementation of the communication strategy will depend on a phased approach. The diagram illustrates the need for short, medium, and long-term communication objectives. RHVP communication activity needs to reflect this in its planning and implementation. Short-term communication activity will ensure effective positioning of RHVP within the region. In effect, this has already begun with the initial website and leaflet. However, this phase should be extended into the next quarter and relevant communication mechanisms used to ensure RHVP has begun to establish a presence in the region and stakeholders are aware the programme is operating. In the medium term ( $3-9$ months $)$, the focus is very much on delivering well-packaged information out to stakeholders. Longer-term objectives focus on behavioural and policy change driven by advocacy work that changes people's perception about alternatives to food aid and moves them to a position of belief, commitment, and action.

RHVP should measure outcomes, changes in opinion, attitudes, and behaviours to determine the effectiveness of persuasive efforts.

The specific objectives of the strategy are to:

maximise impact by implementing a range of communication vehicles that take account of the different communication environments stakeholders operate in/ how different stakeholders engage with or respond to different communication vehicles; ensure a wide scope of stakeholders are targeted with different weighting at different times (informing - knowledge understanding and awareness);

ensure the outcomes of studies/ research relevant to the programmes thematic areas are communicated to different stakeholders in a robust way (includes fast-track studies); and create fertile ground for RHVP advocacy work.

\section{Stakeholders}

Communication activity needs to target a range of players involved in or affected by food insecurity locally, nationally, regionally and internationally: 


\section{Table 1 Players affected by food insecurity}

\section{Table 2 Communication vehicles to achieve change}

\section{Approach and methodology}

identification of key communication vehicles in each country including relevant media, incountry publications etc; identification of key regional stakeholders; contacting regional and international organisations to identify relevant internal communication vehicles; developing concepts for the different products and testing out with all; there will be one strategy across all countries, but for example with tailored lists of mass media, national publications etc; may therefore be helpful to produce matrix of communication activity with each CC; and approach to monitoring and evaluation communication activity to be developed.

Key processes and products

A range of communication vehicles will be developed to meet the communication needs of different stakeholders.

\section{Media Relations}

The management of media relations entails much more than the dissemination of information. The focus in media liaison should be on the management of the corporate image and positive public perceptions of the institution.

To thoroughly understand media liaison it is of utmost importance to understand nature and role of perceptions in media communication (a perception can be regarded as a positive or negative mindset that a person forms about other individuals, institutions, or events. This mental impression influences how he/she feels, thinks and behaves towards those individuals and institutions, or evaluates and judges a specific event).

When dealing with the media, always keep the target audiences of the different media in mind. Media training for relevant functionaries and senior officials and good interpersonal relationships with journalists are a necessity.

\section{Web site}

It is important to realise that a Web site has become far more than a marketing, sales, or communication tool. It is not a huge brochure, a super toll-free line, a product catalogue, or a virtual help desk. With all the possibilities technology offers today, RVHP's website should be the actual organisation - only on the Internet. It is often much easier for a reporter to get information, for a public leader to inquire about the organisation's stance on various issues, for a donor to get financial data on the organisation's website etc. For RVHP, providing all these possibilities online should be most cost effective and efficient.

\section{E-mail}


RHVP needs to build a relationship of trust with its media contacts. E-mail facilitates easier editing of material. It is easier to cut and paste information than it is to take notes and then formulate text, and it is easier than working form a fax or other hard copy. E-mail can carry pictorial material that can be incorporated to illustrate stories.

\section{Radio}

Radio interviews are usually three minutes long, so brevity is essential. Longer, in-depth features, covering personalities and news events, are also presented. Advance contact with the programme organiser or editor by letter, fax, or telephone will normally elicit interest and result in a visit to the studios. News reporters will often visit clients in their business environment. The most common and serious mistake people often make is to bring the wrong person to the studio for an interview. The fact that someone is a senior position does not necessarily mean that he or she is a good communicator. Preparation well beforehand is essential. Studio time is strictly allocated and there is no time then for preparation or deliberation. RHVP are warned against suggesting the line of questioning that the radio interview should follow. However, handing a brief resume of the information to be discussed to the interviewer often prepares the ground for constructive interviews. As with print media, the formula for success is to know the needs of the programme editor or producer, to develop the right personal contacts, and to produce the 'goods' as and when required.

\section{Advertorials}

Given the way the media operate with deadlines and limited space and airtime, RHVP's story may be told in bits and fragments over a prolonged period of time, making it difficult for the public to grasp the 'total story'. It may, therefore be useful to consider developing an information advertisement (advertorial) in a local or national newspaper to ensure that the public is provided with the complete story in one place, from a single source.

To be sure, the release will be used to best advantage it should start with an attention grabbing statement. It should open with the main points of the story by starting who did what, where, when and why. The contact person listed on the release must be prepared to discuss the release with the media and perhaps to be formally interviewed.

\section{Print media}

This research is focusing on newspapers and magazines in South Africa. This kind of research should be done in each of the six countries where RHVP operates. A valuable database will then be established for future media targeting.

\section{Illiterate public}

Although mass media are necessary as carriers of development messages, they alone are not sufficient. The integration of traditional communication and modern mass media, as well as the active participation of people in the development process, is necessary. Outdoor advertising as part of print media is really the only mass advertising medium available, apart from radio. Outdoor advertising provides an increasing 
opportunity for communicating with Third World countries. Radio is the best form of mass media for the rural areas, not only has a massive reach but it is also enjoys a staggering high credibility among its audience (Terblanche 2000: 26).

According to Barker (2001: 9), in Africa, with the high illiteracy and diversity of mothertongue languages, industrial theatre, and road shows as high-impact, cost-effective communication mediums, are a solution that works because it:

taps into the cultural framework of the participants; provides an emotional and then a cognitive experience; facilitates recollection of relevant messages; engages the audience; and utilise everyday situations. The tradition of using song, dance, mime, and theatre to transfer learning (vernacular communication through theatrical techniques), is as old as the history of civilisation. In Africa, this tradition is deeply rooted in the culture of its people (Skinner \& Von Essen 1999: 401). Traditional folk media are cultural resources that accumulate indigenous knowledge - experiences and expressions passed down from generation to generation (Barker 2001: 11).

Folk media is the expressions of cultural lifestyles, consists of a number of forms such as folk theatre, puppet shows, oratory, folk dances, ballads and mime. Traditionally, folk media fulfilled an important entertainment and religious role. In addition, different folk media is also designed by local groups to help in their struggle for a better life (Jayaweera 1987: 17).

The advantages of using folk media in the development process according to Jayaweera (1987: 17) are: folk media is part and parcel of the rural environment and is a credible source of information;

folk media can be seen as so-called living media and is a good example of two-way communication;

themes of folk media are usually flexible and lend themselves to the incorporation of new messages;

folk media is relatively cheap when compared to mass media and

folk media represents actuality and consists of an inexhaustible variety of forms and themes.

Table 3 outlines a suite of communication vehicles that RHVP will produce.

\section{In conclusion: methods of evaluating efforts}

\section{Measurement of Message Exposure}

Message exposure can be measured using several criteria, including the compilation of: press clippings and radio-television mentions; the media impressions, or potential audience reached; Internet hits on the Web site; advertising equivalency, calculated by converting news stories to the cost of a comparable amount of paid space; systematic tracking by usage of computer databases; requests for additional information, often through a toll-free telephone number; and audience attendance at special events. Exposure can also be evaluated by determining the cost to reach each member of the target audience.

\section{Measurement of Audience Awareness}


The next level of evaluation is whether the audience became aware of and understood the message. Audience awareness can be measured with survey research.

\section{Measurement of Audience Action}

Ultimately, public relations campaigns are evaluated by how they help the organisation achieve its objectives through change in audience behaviour, whether this involves sales, fundraising, or the election of a candidate.

\section{Measurement of Supplemental Activities}

A yearly communication audit is necessary to ensure that all publics are receiving appropriate messages. Pre-testing a public relations effort can be done with a pilot test, a split-message approach, a perception analyser system, or the think-aloud method. Meeting and event attendance can be measured both by the number of attendees and by their behaviour, which is an indicator of their acceptance of a message. Newsletter readership can be evaluated by content analysis, interest surveys, and article recall.

\section{ACKNOWLEDGMENTS}

This framework was drawn up for the Regional Hunger and Vulnerability Programme (RHVP) in order to assist Khanya-aicdd (African Institute for Community-Driven Development) to identify key target groups, mechanisms, anticipated impact and other relevant communication concerns or issues.

Initial information on RHVP was obtained in briefing/planning meetings during the period 19 - 27th January 2006, with James Carnegie and Rachel Searle-Mbullu from Khanya-aicdd. Additional information was also obtained and incorporated during informal discussions with colleagues.

*This paper was delivered at SACOMM 2007 - The African Landscape, held in Bloemfontein, 5-7 September.

\section{REFERENCES}

Barker, R. 2001. Communication with communities: A South African experience. Communicatio 27(1): 3-13.

Jayaweera, N. 1987. Rethinking development communication. Singapore: AMIC. Skinner, C. \& Von Essen, L. 1999. Handbook of Public Relations. Cape Town: Oxford Press. Skinner, C., Von Essen, L. \& Mersham, G. 2005. Handbook of Public Relations. Cape Town: Oxford Press.

Terblanche, L. 2006. Communication for Sustainable Agriculture: Study guide for MVL733. Bloemfontein. University of the Free State.

Terblanche, L. 2000. Formulating messages for illiterate rural communities. Communitas 5: 19-32.

Terblanche, L. 2006. Media Management: Study guide for DIM705. Bloemfontein. University of the Free State. 